\title{
XLIII. On M. Jacobi's theory of elliptic functions
}

\section{Rev. Brice Bronwin}

To cite this article: Rev. Brice Bronwin (1843) XLIII. On M. Jacobi's theory of elliptic functions , Philosophical Magazine Series 3, 22:145, 258-262, DOI: 10.1080/14786444308636373

To link to this article: http://dx.doi.org/10.1080/14786444308636373

册 Published online: 01 Jun 2009.

Submit your article to this journal 준

Џll Article views: 4

Q View related articles $₫$ 


\section{The Rev. Brice Bronwin on M.Jacobi's Theory}

mitted to remark, that several interesting inquiries of a kindred nature are suggested by them: the prosecution of these I propose to publish in $\approx$ distinct form, as a supplement to the volume already referred to.

J. R. Young.

XLIII. On M. Jacobi's Theory of Elliptic Functions. By the Rev. Brice Bronwin*.

IN page 36 of his Fundamenta Nova, \&c., M. Jacobi, making $\omega=\frac{m \mathrm{~K}+m^{\prime} \mathrm{K}^{\prime} \sqrt{-1}}{n}$, says that $m$ and $m^{\prime}$ may be any

integer numbers, positive or negative, provided they have no common factor, which also measures $n$. What $I$ intend in this paper is, to prove that $m$ must be an odd and $m^{\prime}$ an even number, and that no other form is admissible. If $r$ and $r^{\prime}$ be integers, positive or negative, the value of $\omega$, as above defined, includes the four following forms:-

$\omega=\frac{(2 r+1) \mathrm{K}+2 r^{\prime} \mathrm{K}^{\prime} \sqrt{-1}}{n}, \sin a m n \omega= \pm 1, \cos a m n \omega=0$.

$\omega=\frac{2 r \mathrm{~K}+2 r^{\prime} \mathrm{K} \sqrt{-1}}{n}, \sin a m n \omega=0, \cos a m n \omega= \pm 1$.

$\omega=\frac{2 r \mathrm{~K}+\left(2 r^{\prime}+1\right) \mathrm{K}^{\prime}}{n} \sqrt{-1}, \sin a m n \omega= \pm \infty \sqrt{-1}, \cos a m n \omega= \pm \infty$.

$\omega=\frac{(2 r+1) \mathrm{K}+\left(2 r^{\prime}+1\right) \mathrm{K}^{\prime} \sqrt{-1}}{n}, \sin a m n \omega= \pm \frac{1}{k}, \cos a m n \omega= \pm \frac{k^{\prime}}{k} \sqrt{-1}$.

The values of $\sin a m n \omega, \cos a m n \omega$ are arnexed on account of their importance in what follows. They are calculated by the formulæ at pages 32 and 34. The references here made are all to the Fundamenta Nova, and the notation adopted there is employed here, unless express mention be made to the contrary. But to abridge I shall write $\mathrm{s} a u$ for $\sin a m u, \mathrm{c} a u$ for $\cos a m u, \& \mathrm{c}$.

At page 38 we have for the general transformation, $\mathrm{s} a \frac{u}{\mathrm{M}}=\mathrm{Cs} a u \mathrm{~s} a(u+4 \omega) \mathrm{s} a(u+8 \omega) \ldots \mathrm{s} a(u+4(n-1) \omega), .(1$. or

$\mathrm{s} a \frac{u}{\mathrm{M}}=\frac{\frac{x}{\mathrm{M}}\left(1-\frac{x^{2}}{\mathrm{~s}^{2} a 4 \omega}\right)\left(1-\frac{x^{2}}{\mathrm{~s}^{2} a}\right) \cdots\left(1-\frac{x^{2}}{\left(1-k^{2} x^{2} \mathrm{~s}^{2} a 4 \omega\right)\left(1-k^{2} x^{2} \mathrm{~s}^{2} a 8 \omega\right) \ldots\left(1-k^{2} x^{2} \mathrm{~s}^{2} a 2(n-1) \omega\right.}\right)}{(n-1) \omega)}$.

Here $\frac{1}{C}$ is put for the constant denominator of the second member of (1.) The middle factor of that member is * Communicated by the Author. 
$\mathrm{s} a(u+2(n-1) \omega)$, and the following factors easily reduce to $\pm \mathrm{s} a(u+2 \omega), \pm \mathrm{s} a(u+6 \omega)$, \&c., whatever form $\omega$ takes. Also in (2.) $\mathrm{s} a(2 n-2), \mathrm{s} a(2 n-6 w)$, \&c. reduce to $\pm \mathrm{s} a 2 \omega, \pm \mathrm{s} a 6 \omega, 8 \mathrm{c}$. Therefore (1.) and (2.) reduce to $\mathrm{s} a \frac{u}{\mathrm{M}}=\mathrm{Cs} a u \mathrm{~s} a(u+2 \omega) \mathrm{s} a(u+4 \omega) \ldots \mathrm{s} a(u+2(n-1) \omega)$. (3.)

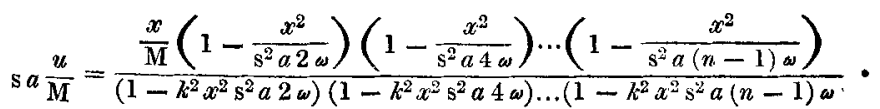

The quantity $\mathrm{C}$, if we give it M. Jacobi's form, will reduce in like manner; but this is of no consequence. M. Jacobi appears to have been aware of the reduction above effected, as he has partially made it at pages 41 and 51 .

It hence appears that the second form in (A.) will always reduce to one of the three other forms. For making $\omega^{\prime}$ $=\frac{r \mathrm{~K}+r^{\prime} \mathrm{K}^{\prime} \sqrt{-1}}{n}$, we have $\omega=2 \omega^{\prime}$; and putting $2 \omega^{\prime}$ for $\omega$ in (3.) and (4.), these last become of the form (1.) and (2.); which will again reduce to the forms (3.) and (4.), $\omega^{\prime}$ being in the place of $\omega$. And if $\omega^{\prime}$ be divisible by 2 , we may repeat the operation, and may continue to do so till we arrive at an ${ }_{\omega}^{\prime \prime \prime}=\frac{p \mathrm{~K}+q \mathrm{~K}^{\prime} \sqrt{-1}}{n}$, in which one or both the quantities $p$ and $q$ are odd numbers. The same reduction might also be made in the values of $\mathrm{c} a \frac{u}{\mathrm{M}}$ and $\Delta a \frac{u}{\mathrm{M}}$. When, therefore, $\infty$ is divisible by 2 , the formulæ of transformation will reduce till $\omega$ takes one of the three other forms.

Again, the second members of (1.) and (2.) are not proper representations of the first. For since saz $(n-1) \omega= \pm s a 2 \omega$, $\mathrm{s} a 2(n-3) \omega= \pm \mathrm{s} a 6 \omega, 8 \mathrm{c}$.; these members vanish when $u=2 \omega, u=6 \omega, \& c$, and therefore ought to contain the factors $1-\frac{x^{2}}{s^{2} a 2 \omega}, 1-\frac{x^{2}}{s^{2} a 6 \omega}$, \&c. It must be remembered that $x=\mathrm{s} a u$. For the same reason the second members of (3.) and (4.) would be improper representations of the first, if w were divisible by 2 . The second form of (A.) therefore is inadmissible.

For the three other forms of $\omega$, we cannot reduce $s a(n-1) \omega$ to $\pm \mathrm{s} a \omega, \mathrm{s} a(n-3) \omega$ to $\pm \mathrm{s} a 3 \omega, \& \mathrm{c}$; ; and it is not possible to reduce them to sines of any other amplitudes. For these values of $\omega$, then, (3.) and (4.) are in their simplest forms; their second members vanish when $u=0,2 \omega, 4 \omega, \& c$., but never 
260 The Rev. Brice Bronwin on M. Jacobi's Theory

between these values. Consequently, while $u$ increases of $2 \omega$, $\frac{u}{\mathrm{M}}$ increases of $2 \mathrm{H}$, neither more nor less, if $\frac{u}{\mathrm{M}}=\mathrm{H}$ when its amplitude is $\frac{\pi}{2}$. Whilst, therefore, $u$ from 0 becomes $\omega, \frac{u}{\mathrm{M}}$ from 0 becomes $H$. Let them have these values in (3.), and we obtain $\mathrm{s} a \mathrm{H}=1= \pm \mathrm{Cs} a \omega \mathrm{s} a 3 \omega \ldots . . \mathrm{s} a(2 n-1) \omega$. Therefore

$$
\frac{1}{\mathbf{C}}= \pm \mathrm{s} a \omega \mathrm{s} a 3 \omega \mathrm{s} a 5 \omega \ldots \ldots \mathrm{s} a(2 n-1) \omega . \quad .
$$

This then is the general form of $\frac{1}{\mathrm{C}}$, and M. Jacobi's denominator cannot be true, except in those cases in which it is reducible to it.

One factor of (5.) is $\operatorname{san} \omega$. The second and third forms (A.), therefore render $\mathrm{C}$ faulty, and also the values of $\mathrm{s} a \frac{u}{\mathrm{M}}$, of $\mathrm{M}$, and of the modulus of $\frac{u}{M}$ faulty; for $C$ enters into these values. If M. Jacobi's formulæ do not fail for these values of $\omega$, it is because they do not hold true for them. His value of $\frac{1}{C}$ is

$$
\begin{gathered}
\{\mathrm{s} a(\mathrm{~K}-4 \omega) \mathrm{s} a(\mathrm{~K}-8 \omega) \ldots \ldots \mathrm{s} a(\mathrm{~K}-2(n-1) \omega)\}^{2} \\
=\left\{\frac{\mathrm{c} a 4 \omega \mathrm{c} a 8 \omega \ldots \ldots \mathrm{c} a 2(n-1) \omega}{\Delta . a 4 \omega \Delta . a 8 \omega \ldots . . \Delta . a 2(n-1) \omega}\right\}^{2} \\
=\left\{\frac{\mathrm{c} a 2 \omega \mathrm{c} a 4 \omega \ldots \ldots \mathrm{c} a(n-1) \omega}{\Delta . a 2 \omega \Delta . a 4 \omega \ldots \ldots \Delta . a(n-1) \omega}\right\}^{2},
\end{gathered}
$$

whatever be the form of $w$. But for the first of the forms (A.) only can we have

$\mathrm{s} a \omega \mathrm{s} a 3 \omega \ldots . . \mathrm{s} a(2 n-1) \omega=\{\operatorname{sa\omega s} a 3 \omega \ldots . . \mathrm{s} a(n-2) \omega\}^{2}$

$$
=\left\{\frac{\mathrm{c} a 2 \omega \mathrm{c} a 4 \omega \ldots \ldots \mathrm{c} a(n-1) \omega}{\Delta . a 2 \omega \Delta . a 4 \omega \ldots \ldots \Delta . a(n-1) \omega}\right\}^{\mathrm{s}} .
$$

For the other forms of $\omega$ this reduction cannot be effected. M. Jacobi's formulæ therefore are only true for the first form (A.) In page 39 we have

$\operatorname{ca} \frac{u}{\mathrm{M}}=\frac{\operatorname{c} a u \mathrm{c} a(u+4 \omega) \mathrm{c} a(u+8 \omega) \ldots \mathrm{c} a(u+4(n-1) \omega)}{\{\mathrm{c} a 4 \omega \mathrm{c} a 8 \omega \ldots \mathrm{c} a 2(n-1) \omega\}^{2}}$.

This reduces as before to

$$
\operatorname{c} a \frac{u}{\mathrm{M}}= \pm \frac{\operatorname{c} a u \operatorname{c} a(u+2 \omega) \operatorname{c} a(u+4 \omega) \ldots \mathrm{c} a(u+2(n-1) \omega)}{\{\operatorname{c} a 2 \omega \mathrm{c} a 4 \omega \ldots \mathrm{c} a(n-1) \omega\}^{2}}
$$


This last must vanish when $u=\omega, 3 \omega, 5 \omega, \& c$., because then $\frac{u}{M}=\mathrm{H}, 3 \mathrm{H}$, \&c. The only factor in the numerator of the second member which can vanish is $\mathbf{c} a n \omega$; but this vanishes only for the first of the forms (A.), which therefore is the only admissible form. Moreover, the second of (A.) makes $\mathrm{s} a \mathrm{H}, \mathrm{s} a \& \mathrm{H}$, \&c. $=0$, which is absurd. The third of (A.) gives a factor infinite in both $\mathrm{s} a \mathrm{H}, \mathrm{c} a \mathrm{H}, 8 \mathrm{c}$.

If we develope (7.), it should give

$c a \frac{u}{\mathrm{M}}=\sqrt{1-x^{2}} \frac{\left(1-\frac{x^{2}}{\mathrm{~s}^{2} a \omega}\right)\left(1-\frac{x^{2}}{\mathrm{~s}^{2} a 3 \omega}\right) \ldots\left(1-\frac{x^{2}}{\left.\mathrm{~s}^{2}-k^{2} x^{2} \mathrm{~s}^{2} a 2 \omega\right)\left(1-k^{2} x^{2} \mathrm{~s}^{2} a 4 \omega\right) \ldots\left(1-k^{2} x^{2} \mathrm{~s}^{2} a(n-1) \omega\right)}\right)}{.} \cdot$

The first formula in page 39, which is the development of (6.), should reduce to (8.), which vanishes for all the odd multiples of $\omega$. But these reductions cannot be effected, except for the first of the forms (A.). If we deduce (8.) immediately from (4.), as Sir James Ivory has done, we find that $\sqrt{1-x^{2}}$ results from $\sqrt{1-\frac{\mathrm{s}^{2} a u}{\mathrm{~s}^{2} a n \omega}}$, which indeed is evident. This excludes all the values of $\omega$ but the first, for this factor must vanish when $x=\mathrm{s} a u= \pm 1$. And hence it is evident that the form of M. Jacobi's theory excludes all other values of $\omega$ but the first.

From what has been done, it is abundantly plain that $\mathbf{M}$. Jacobi's transformation is true only for the form

$$
\omega=\frac{(2 r+1) \mathrm{K}+2 r^{\prime} \mathrm{K}^{\prime} \sqrt{-1}}{n} ;
$$

and, moreover, that if we substitute for $\frac{1}{C}$ the general value (5.) instead of his value, it would fail for all the other forms of $\omega$. The only possible value of $\omega$, therefore, is the preceding; consequently many of the forms suggested at pages 49 and 50 would fail, as $\frac{i \mathrm{~K}^{\prime}}{n}, \frac{\mathrm{K}+i \mathrm{~K}^{\prime}}{n}, \frac{\mathrm{K}+3 i \mathrm{~K}^{\prime},}{n} \frac{2 \mathrm{~K}+i \mathrm{~K}^{\prime}}{n}$, $\frac{3 \mathrm{~K}+i \mathrm{~K}^{\prime}}{n}, \frac{(n-1) \mathrm{K}+i \mathrm{~K}^{\prime}}{n}$, and some others. The particular transformation given in pages $52,53,54,55$ must fail; and as we cannot make $\omega=\frac{m \mathrm{~K}^{\prime} \sqrt{-1}}{n}$, we cannot obtain a real transformation by means of imaginary quantities. Indeed it does not appear that any other form than $\omega=\frac{(2 r+1) \mathrm{K}}{n}$ would be of any utility; and this last, it is easy to see, will 
262 The Rev. Prof. B. Powell on Circularly Polarized Light. reduce to $\omega=\frac{K}{n}$; for the functions of all amplitudes greater than $u+2(n-1) w$ would reduce to similar functions of amplitudes less than this.

In the 15th Number of the Cambridge Mathematical Journal, I very briefly pointed out the failure of some of M. Jacobi's forms for $\omega$. This the editor of that work was very reluctant to admit; and in the 16th Number of the same $I$ find a Note, bearing the signature $\mathrm{C}$., intended as a refutation of what I had advanced. On this Note I must now make a few observations, but after what has been done in this paper it will not be necessary to enter far into particulars.

If the writer wished to compare my denominator with $\mathrm{M}$. Jacobi's, he should, for each particular form of $\omega$, have attempted to reduce them to the same quantity. His method gives the true one for the first value of $\omega$, which proves to be mine, and affords no foundation for the inferences he has drawn from it. The true form would also be ascertained the moment it is proved, that when $u=\omega, v=\mathrm{H}$. And this is the important point; if they differ, and when they differ, which is right. For the second value of $\omega$, the formulæ (1.), (2.) admit of reduction, as already shown. [The references are made to the note.] The third gives a factor infinite in the numerators of the second members of (1.), (2.) when $u=\omega$, and is therefore evidently inudmissible. For the last value of $\omega, \mathrm{s} a n \omega$ $= \pm \frac{1}{k}, \operatorname{c} a n \omega= \pm \frac{k^{\prime}}{k} \sqrt{-1}$; and these values the writer of the note has, evidently by mistake, taken for the values of $\mathrm{s} a v, \mathrm{c} a v$. For surely he never could prove, that when $u=\omega$ the second member of (2.), divided by c $a n \omega$, is equal to unity. His last value of $\mathbf{C}$ therefore is wrong. I feel compelled to say that this Note is perfectly absurd at every step of it; and if the author had proved what he aimed at, and which is really true, it would have been nothing to the purpose.

Denby, near Huddersfield, Jan. 5th, 1843.

XLIV. On Mr. Earnshaw's Deduction of a Property of Circularly Polarized Light. By Professor PoweLn.

To the Editors of the Philosophical Magazine and Journal. GentLemen,

IN a late Number of your Journal there appears a theoretical $I_{\text {deduction by }} \mathrm{Mr}$. Earnshaw to this effect, that if circularly polarized light, right-handed, for example, fall on glass at a 